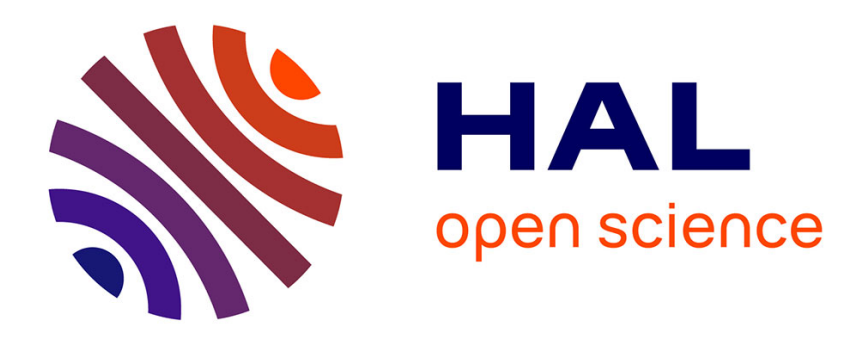

\title{
Medium range interaction between neutral metal atoms and a metallic surface $(\mathrm{Ta} / \mathrm{W})$
}

\author{
J. Bardon, M. Audiffren
}

\section{To cite this version:}

J. Bardon, M. Audiffren. Medium range interaction between neutral metal atoms and a metallic surface (Ta/W). Journal de Physique Lettres, 1983, 44 (21), pp.883-887. 10.1051/jphyslet:019830044021088300 . jpa-00232278

\section{HAL Id: jpa-00232278 https://hal.science/jpa-00232278}

Submitted on 1 Jan 1983

HAL is a multi-disciplinary open access archive for the deposit and dissemination of scientific research documents, whether they are published or not. The documents may come from teaching and research institutions in France or abroad, or from public or private research centers.
L'archive ouverte pluridisciplinaire HAL, est destinée au dépôt et à la diffusion de documents scientifiques de niveau recherche, publiés ou non, émanant des établissements d'enseignement et de recherche français ou étrangers, des laboratoires publics ou privés. 
Classification

Physics Abstracts

79. 20R - 61.16F

\title{
Medium range interaction between neutral metal atoms and a metallic surface $(\mathrm{Ta} / \mathrm{W})$
}

\author{
J. Bardon (*) and M. Audiffren (*) \\ CRMC $^{2}$-CNRS, Campus de Luminy, case 913, 13288 Marseille Cedex 09, France
}

(Reçu le 20 juin 1983, accepté le 13 septembre 1983)

\begin{abstract}
Résumé. - La présence anormale d'adatomes dans la partie cachée d'une pointe d'émission ionique de champ après un dépôt est interprétée par des interactions de van der Waals à moyenne distance (10-30 $\AA)$ entre la pointe et les atomes projetés. Pour la première fois, la constante d'interaction a été mesurée pour des métaux de transition (Ta/W). La valeur obtenue est $18 \mathrm{eV} \AA^{3}$.

Abstract. - The anomalous presence of adatoms in the hidden part of a field ion microscope tip after a sideward deposition is interpreted by the existence of a medium range (10-30 $\AA$ ) van der Waals interaction between the tip and the incoming atoms. For the first time, the interaction constant has been measured between transition metals $(\mathrm{Ta} / \mathrm{W})$. The obtained value is $18 \mathrm{eV} \AA^{3}$.
\end{abstract}

\section{Introduction.}

During experiments using the strong gradient of the electric field near the apex of a field ion microscope tip to measure the polarizability of neutral atoms, we have obtained some unexpected results. The experimental device is described in figure 1 . A source of tantalum atoms is set on the side of a tungsten field ion microscope tip, far enough for the velocities of incoming atoms to be considered as parallel. In the absence of an electric field during the deposition, one can expect that all the Ta atoms would impinge on the "Sunny " part (S-part) of the tip limited by the $x \mathrm{O} z$ plane, so that the field ion image would not reveal any Ta adatom in the shadowed region(D-part). In fact, some adatoms are observed in this D-part.

We interpret this phenomenon by the curvature of the expected trajectory by van der Waals interactions and determine the corresponding $C$ constant. The interest in this interaction is rapidly growing due to its importance for the interpretation of atomic beam scattering but the results actually available concern principally the interaction between rare gases and surfaces [1].

Metal atom-metal surface interactions have been studied only [2-5] for some alkali atoms in the $200 \AA-300 \AA$ range where retarded interaction can be suspected.

(*) Also Université de Provence, U.E.R. de Physique, 13003 Marseille. 


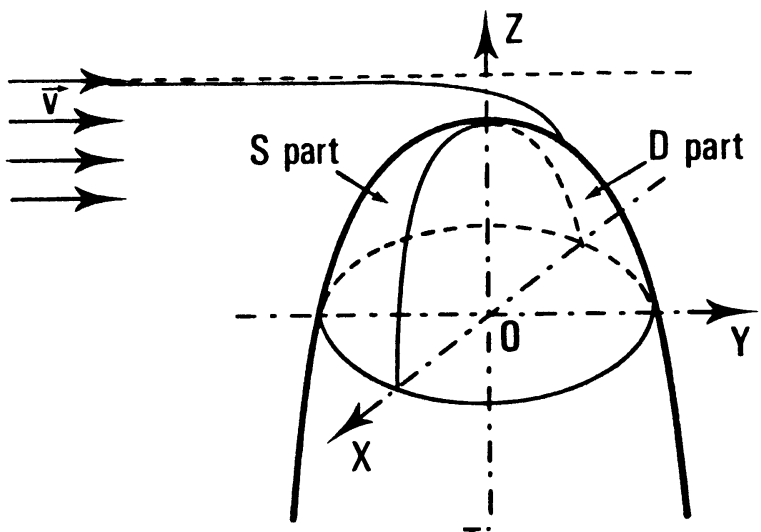

Tip

Fig. 1. - Trajectories of atoms with interaction (full line) and without interaction (dotted line). Without interaction, the atoms incoming with velocities parallel to the $\mathrm{O} y$ axis should strike the tip in the S-part only.

\section{Experimental.}

The field ion microscope is a conventional one, described elsewhere [6]. The background pressure achieved using rigorous procedures [7] is below $2 \times 10^{-11}$ torr, the X-ray limit of the gauge. When the Ta source is activated, the pressure remains within the $10^{-10}$ torr limit.

The source is a DC current heated Ta wire $0.2 \mathrm{~mm}$ in diameter. A four lead monitoring system enables a source temperature of $2900 \mathrm{~K} \pm 15 \mathrm{~K}$ to be obtained. A shield, with a $2 \mathrm{~mm}$ diameter hole, is placed between the filament and the tip and is polarized at $-9 \mathrm{~V}$ with respect to the filament when the source is operated. This shield :

- is a repeller for thermal electrons

- minimizes the thermal energy radiated to the tip

- provides an angular limitation $\left(3^{\circ}\right)$ of the initial atoms velocities.

The number of ions produced by the source is negligible [8].

A clean perfect surface is first produced by field evaporating the tip. Then a reference micrograph of this surface is taken with the tip at $80 \mathrm{~K}$ and at a voltage giving the best image (B.I.V.) with $P_{\mathrm{He}}=10^{-5}$ torr. The imaging gas is then pumped down to $10^{-10}$ torr and the Ta source is activated. During the deposition, the tip temperature is maintained at $180 \mathrm{~K}$.

This temperature is below the Ta adatoms diffusion [9] temperature but high enough to ensure that an atom, possibly arriving at a metastable site, reaches the nearest stable adsorption site. We thus avoid any unwanted field evaporation during the establishment of the image voltage. $10^{-5}$ torr helium pressure is then re-established and three micrographs are taken at $0.7,0.8$ and 0.9 of the B.I.V. This procedure is mandatory because some adatoms are field evaporated before other ones become visible.

These three micrographs are compared to the reference micrograph by the colour superposition method [10], giving the number and the location of the atoms that have reached the tip. The colour superposition method allows us to ascertain that : (i) no contamination occurs during the experiment, (ii) no surface atom displacement is induced by the impinging atoms and (iii) no accidental tip heating by the radiation from the source occurs during successive depositions.

A number of patterns like that of figure 2 is obtained by this method in which $N_{\mathrm{s}}$ adatoms are located and counted in the S-part and $N_{\mathrm{D}}$ in the D-part. A difficulty is the location of the L line, the boundary between the two regions, due to the geometrical uncertainty on the angular position of the tip. To solve this problem, a second source was disposed symmetrically with respect to the 


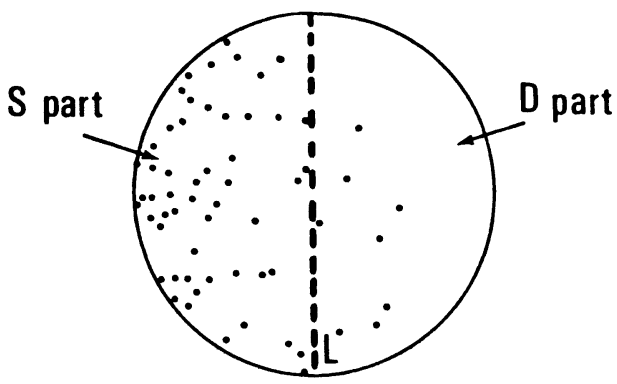

Fig. 2. - Example of a pattern corresponding to one deposition. Each dot corresponds to an adatom.

tip. The alignment of the two sources and the tip was easily adjusted optically within two degrees. Thus the L line can be located at the crossing of the statistical $N_{\mathrm{D}} / N_{\mathrm{S}}$ curves of both sources.

\section{Results.}

By graphically superimposing patterns like that of figure 2, we did not observe any abnormal accumulation or lack of adatoms at any place. This should have occurred in the case of a geometrically defective tip or if there were a significant anisotropy in the interaction between the surface and the incoming atoms.

Summed over 39 depositions from the two sources, the number of adatoms counted in the D-part was $N_{\mathrm{D}}=133$, and in the S-part $N_{\mathrm{S}}=1,128$. The statistical uncertainty on the ratio $N_{\mathrm{D}} / N_{\mathrm{S}}$ is less than ten percent.

\section{Discussion.}

Adatoms in the D-part have been observed by Gurney et al. [11] who explained this phenomenon by the backscattering of the atoms by the walls of the system. To check this assumption, we shielded the tip from the direct beam with a narrow $(0.5 \mathrm{~mm}$ wide $)$ metal foil. With this arrangement, we never observed any adatom in the image. Therefore, the above mentioned hypothesis can be discarded in view of the negligibly small influence of the shield on the hypothetical backscattering.

A similar observation was first made by Ehrlich [12]. He interpreted the result by a skating process : the incoming atom making several hops before its kinetic and adsorption energies are completely exchanged with the substrate. This mechanism was sustained by a one-dimensional ball and spring model [13].

Such a hopping process probably occurs in numerous cases of adsorption of gases on metals. However, as pointed by King [14], it gives a non random distribution of the adatoms on the surface because of their lateral interactions. Also Ehrlich [12] remarks that this hopping should give an accumulation of adatoms on the ledges of a stepped surface like a field ion tip. Convincing evidence was given by Young and Schubert [15] that a random distribution is obtained in the case of tungsten atoms and tungsten surface, which made them conclude that the atom remains in the first potential well encountered. Similarly, a careful examination of our micrographs for tantalum and tungsten on tungsten shows that the number of adatoms on the terraces versus the ledges corresponds to a random distribution.

The mechanism of energy transfer between an adsorbing atom and a surface is a subject of current interest. It seems that electron hole excitations have to be considered simultaneously with phonon creation in the case of an adsorbate with bound states [16]. The earlier calculation of McCarrol and Ehrlich [13] on a linear ball and spring model could therefore be unsuited in the 
case of tantalum (or tungsten) on a tungsten surface. We can thus conclude that the skating process is not responsible for the presence of the adatoms in the D-part of the tip.

One can also imagine a hard ball model [17] where an atom striking one end of a nearest neighbour row would destabilize the atom at the other end. The presence of adatoms on the (110) plane and the low evaporation field, characterising Ta adatoms, are not consistent with this hypothesis.

In the case of interactions between metals, the van der Waals interaction is usually neglected when compared with the strong chemical interaction. Lifschitz [18] and Zaremba and Kohn [19] have demonstrated that $W$, the interaction energy between an atom and a metallic surface is $W=-C / Z^{3}$ (Eq. 1) where $C$ is a constant and $Z$ the distance between the atom and the surface.

We have calculated, by computer simulation of the trajectories, the ratio $N_{\mathrm{D}} / N_{\mathrm{S}}$, taking into account the geometrical configuration of the system and the Maxwellian distribution of the velocities of Ta atoms for different values of $C$. The imaged part of the tip was approximated by a sphere of radius equal to the curvature radius of the tip. Equation 1 has been established in the case of a plane surface, but the ratio of the typical interaction distance $(20 \AA)$ to the radius of the tip is about 0.1 and permits the assumption that the sphere approximates to a plane.

As demonstrated by Mehl and Schaich [20], because of the short range of the interaction, one should use a quantum mechanical/calculation of the trajectories. However, they showed that classical and fully quantum mechanical calculations give the same result within an extended range of validity.

The value of $C$ giving a value of $N_{\mathrm{D}} / N_{\mathrm{S}}$ equal to our experimental result is

$$
C=18 \mathrm{eV} . \AA^{3} \text {. }
$$

The theoretical [11] value of this constant is

$$
C=\int_{0}^{\infty} \alpha(i u) \cdot \frac{\varepsilon(i u)-1}{\varepsilon(i u)+1} \mathrm{~d} u
$$

where $\alpha(i u)$ is the polarizability of the atom and $\varepsilon(i u)$ the dielectric function of the solid, both continued to imaginary frequencies. A straightforward calculation of $C$ is beyond the scope of this paper due to the complex spectrum of tantalum where LS coupling gives infrared absorption and to the lack of data for the dielectric function of tungsten below $1 \mathrm{eV}[21,22]$. Detailed calculations should also take into account the small dimension of the tip which cannot be considered as a semi-infinite solid.

It is noticeable that the value of $C$ which we have obtained is 100 times the value [19] for the interaction between noble metals and helium. The static polarizability of tantalum, obtained theoretically [23] and roughly confirmed by our preliminary experimental results, is also 100 times greater than the static polarizability of He. We think that this agreement, concerning the order of magnitude, may be due to the smoothness of the function $\alpha(i u)$ at low energies. Such a proportionality between $C$ and the static polarizability has also been observed [1] in the case of rare gas and dielectric surfaces. We can therefore reasonably suppose that the curvature of the trajectories by van der Waals forces is responsible for the presence of adatoms in the D-zone.

The estimated accuracy on the value of $C$ is about 30 percent due the statistical uncertainty of the measurements and the non absolute sphericity of the tip.

\section{Conclusion.}

As already observed by other authors, during a deposition, some atoms arrive at the geometrically hidden part of a field ion microscope tip. We have interpreted this phenomenon by the van der Waals force. We have also, for the first time, measured the van der Waals interaction between 
Ta atoms and a metallic surface, the value obtained for the interaction constant is $C=18 \mathrm{eV} . \AA^{3}$. This work demonstrates that the van der Waals forces, usually neglected in the case of metallic interactions, can play an important rôle in some experiments. Measurements are in progress for other transition metals.

The unique capability of the field ion microscopy to observe atomic events and to measure very small displacements is very well adapted to the study of such weak interactions.

\section{Acknowledgments.}

The authors very much appreciated helpful discussions with Dr R. Morin and Dr J. Bermond throughout the investigation. They also thank Pr M. Drechsler for his constant interest in the development of this work.

\section{References}

[1] Hoinkes, H., Rev. Mod. Phys. 52 (1980) 933.

[2] Raskin, D., Kush, P., Phys. Rev. 179 (1969) 712.

[3] Shih, A., Raskin, D., Kush, P., Phys. Rev. A 9 (1974) 652.

[4] Shin, A., Phys. Rev. A 9 (1974) 1507.

[5] Shih, A., Parsegian, V. A., Phys. Rev. A 12 (1975) 835.

[6] Audiffren, M., Traimond, P., Bardon, J. and Drechsler, M., Surf. Sci. 75 (1978) 751.

[7] Ayrault, G. and Ehrlich, G., J. Chem. Phys. 601 (1974) 281.

[8] SCHEER, M. D. and Fine, J., The Structure and Chemistry of Solid Surfaces, Somorjai ed. (J. Wiley and Sons, Inc., New York) 1969.

[9] Bassett, D. W., Parsley, M. J., J. Phys. D 3 (1970) 707.

[10] Muller, E. W. and Tsong, T. T., Field Ion Microscopy (Elsevier, Amsterdam) 1969.

[11] GuRney, T., Hutchinson, F., Young, R. D., J. Chem. Phys. 4211 (1965) 3939.

[12] Ehrlich, G., in Metal Surfaces (ASM, Metals Park, Ohio) 1963.

[13] McCarroll, B., Ehrlich, G., J. Chem. Phys. 382 (1963) 523.

[14] KING, D. A., Proc. 7th Intern. Vac. Congr. and 3rd Intern. Conf. Solid Surfaces (Vienna) 1977.

[15] Young, R. D. and Schubert, D. C., J. Chem. Phys. 42 (1965) 3943.

[16] e.g. Nourtier, A., J. Physique 38 (1977) 479.

NoRsKov, J. K., LuNDOPVIST, B. I., Surf. Sci. 89 (1979) 251.

Schönhanner, K., Gunnarson, O., Phys. Rev. B 224 (1980) 1629.

Gunnarson, O., Schönhammer, K., Phys. Rev. B 254 (1982) 2503.

INGLESFIELD, J. E., Surf. Sci. 127555.

[17] DreChSLER, M., Private Communication.

[18] LifschitZ, E. M., Zh. Eksp. Teor. Fiz 29 (1955) 94.

[19] Zaremba, E. and KoHn, W., Phys. Rev. B 136 (1976) 2270.

[20] Mehl, M. J., Schaich, W. L., Phys. Rev. A 214 (1980) 1177.

[21] Weaver, J. H., Olson, G. and LyNCH, D. W., Phys. Rev. B 12 (1975) 1293.

[22] Gravier, P., Thesis, Marseilles 1979, Unpublished.

[23] Teachout, R. R. and PaCk, R. T., Atomic Data 3 (1975) 195. 